\title{
A remarkably stable TipE gene cluster: evolution of insect Para sodium channel auxiliary subunits
}

\author{
Jia Li ${ }^{1,2}$, Robert M Waterhouse ${ }^{1,2}$ and Evgeny M Zdobnov ${ }^{1,2,3^{*}}$
}

\begin{abstract}
Background: First identified in fruit flies with temperature-sensitive paralysis phenotypes, the Drosophila melanogaster TipE locus encodes four voltage-gated sodium ( $\mathrm{Nav}_{\mathrm{v}}$ ) channel auxiliary subunits. This cluster of TipE-like genes on chromosome $3 \mathrm{~L}$, and a fifth family member on chromosome 3R, are important for the optional expression and functionality of the Para $\mathrm{Na}_{v}$ channel but appear quite distinct from auxiliary subunits in vertebrates. Here, we exploited available arthropod genomic resources to trace the origin of TipE-like genes by mapping their evolutionary histories and examining their genomic architectures.

Results: We identified a remarkably conserved synteny block of TipE-like orthologues with well-maintained local gene arrangements from 21 insect species. Homologues in the water flea, Daphnia pulex, suggest an ancestral pancrustacean repertoire of four TipE-like genes; a subsequent gene duplication may have generated functional redundancy allowing gene losses in the silk moth and mosquitoes. Intronic nesting of the insect TipE gene cluster probably occurred following the divergence from crustaceans, but in the flour beetle and silk moth genomes the clusters apparently escaped from nesting. Across Pancrustacea, TipE gene family members have experienced intronic nesting, escape from nesting, retrotransposition, translocation, and gene loss events while generally maintaining their local gene neighbourhoods. D. melanogaster TipE-like genes exhibit coordinated spatial and temporal regulation of expression distinct from their host gene but well-correlated with their regulatory target, the Para Nav channel, suggesting that functional constraints may preserve the TipE gene cluster. We identified homology between TipE-like $\mathrm{Na}_{v}$ channel regulators and vertebrate Slo-beta auxiliary subunits of big-conductance calcium-activated potassium $\left(B_{C_{2}}\right)$ channels, which suggests that ion channel regulatory partners have evolved distinct lineage-specific characteristics.

Conclusions: TipE-like genes form a remarkably conserved genomic cluster across all examined insect genomes. This study reveals likely structural and functional constraints on the genomic evolution of insect TipE gene family members maintained in synteny over hundreds of millions of years of evolution. The likely common origin of these $\mathrm{Nav}$ channel regulators with $\mathrm{BK}_{\mathrm{Ca}}$ auxiliary subunits highlights the evolutionary plasticity of ion channel regulatory mechanisms.
\end{abstract}

\section{Background}

Voltage-gated sodium $\left(\mathrm{Na}_{\mathrm{V}}\right)$ channels control the passage of sodium ions across the plasma membrane and play a crucial role in the creation and propagation of electrical signals in excitable cells, such as neurons and myocytes. The pore-forming alpha subunits comprise a gene family of up to ten members in vertebrate species [1], which are associated with four types of auxiliary

\footnotetext{
* Correspondence: Evgeny.Zdobnov@unige.ch

'Department of Genetic Medicine and Development, University of Geneva

Medical School, rue Michel-Servet 1, 1211 Geneva, Switzerland

Full list of author information is available at the end of the article
}

beta-subunits that are important for the biogenesis and gating kinetics of functional alpha-subunits [2]. In contrast, only two $\mathrm{NaV}$ channel alpha-subunit genes are found in insects, orthologues of Drosophila melanogaster DSC1 (Drosophila sodium channel 1) and Para (paralytic), and sequence homology searches fail to identify homologues of the vertebrate $\mathrm{Na}_{\mathrm{V}}$ channel auxiliary beta-subunits. Although DSC1 may rather function as a calcium channel involved in olfactory avoidance responses [1,3,4], Para mutants exhibit temperature-sensitive paralysis caused by loss of $\mathrm{Na}^{+}$-based action potentials [5-8]. Thus, insect genomes appear to encode
C Biomed Central 
only one functional $\mathrm{Na}_{\mathrm{V}}$ channel alpha-subunit gene and no orthologues of the vertebrate $\mathrm{Na}_{\mathrm{V}}$ channel auxiliary beta-subunits.

Studies of temperature-sensitive paralysis phenotypes in D. melanogaster identified a role for the TipE locus (temperature-induced paralysis locus E) in cell excitability, and suggested regulatory interactions with the Para $\mathrm{Na}_{V}$ channel $[9,10]$. Subsequent identification of the TipE gene facilitated studies that demonstrated its $\mathrm{Na}_{\mathrm{V}}$ channel regulator activity by stimulating functional expression of Para in Xenopus oocytes, and established that TipE expression during pupal development rescued the paralysis phenotype in adult fruit flies [11]. Moreover, sodium-dependent repetitive firing in Drosophila neurons is regulated by TipE [12], and Para-TipE interactions influence the pharmacology of the Para $\mathrm{Na}_{\mathrm{V}}$ channel [13]. Despite the lack of insect orthologues of vertebrate $\mathrm{Na}_{\mathrm{V}}$ channel auxiliary beta-subunits, characterization of the D. melanogaster TipE gene revealed strikingly similar functions: an auxiliary subunit required for the correct assembly and gating of the Para $\mathrm{Na}_{\mathrm{V}}$ channel. Furthermore, four homologues of TipE (Teh14 ) are encoded in the $D$. melanogaster genome and their TipE-like functions as auxiliary subunits of Para $\mathrm{Na}_{\mathrm{V}}$ channels have been characterized through co-expression with Para in Xenopus oocytes [14]. Thus, fruit fly TipE gene family members are important for the optimal expression and functionality of the Para $\mathrm{Na}_{\mathrm{V}}$ channel but appear quite distinct from auxiliary beta-subunits in vertebrates.

Their key roles in membrane excitability make insect $\mathrm{Na}_{\mathrm{V}}$ channels attractive targets for the development of insecticides $[13,15,16]$. However, Para mutations lead to knockdown resistance in insects and other arthropods, which reduces the efficacy of many insecticides and intensifies the search for alternative targets [17-19]. Characterizing the evolutionary histories and molecular functions of insect TipE gene family members will not only further the understanding of the mechanisms controlling cell excitability, but may also offer novel opportunities for developing alternative insect control strategies. In this study, we examined the genealogies of TipE gene family members across multiple insect species with sequenced genomes, and revealed a remarkably conserved synteny block of TipE homologues with local gene arrangements that have been well-maintained in many insect genomes over hundreds of millions of years of evolution.

\section{Results and discussion}

A genomic cluster of TipE gene family members conserved across Insecta

High levels of genome shuffling observed in many insect species lead to the rapid decay of synteny, leaving only a few identifiable conserved gene clusters [20]. Examples of such well-maintained local gene arrangements include the homeobox (Hox) [21] and wingless (Wnt) [22] gene clusters with key roles in early development. Synteny block analysis across 23 arthropod species with sequenced genomes (see Methods) identified both Hox and Wnt gene clusters, and highlighted a genomic cluster of TipE gene family members as one of the most conserved blocks of orthologues with well-maintained local gene arrangements. This highly conserved synteny block was defined by a core of three sets of orthologous genes from 20 insect species: Drosophila melanogaster and 11 other fruit flies; three mosquitoes: Anopheles gambiae, Aedes aegypti, and Culex quinquefasciatus; the silk moth, Bombyx mori; the flour beetle, Tribolium castaneum; the parasitoid wasp, Nasonia vitripennis; the honey bee, Apis mellifera; and the human body louse, Pediculus humanus. Sequence homology searches of genomic resources of the remaining sequenced arthropod species revealed a similarly conserved gene arrangement in the pea aphid, Acyrthosiphon pisum, as well as four TipE homologues in the crustacean water flea, Daphnia pulex, but no homologous genes in the arachnid deer tick, Ixodes scapularis (possibly due to incomplete genome sequencing). The three D. melanogaster genes are located in a $\sim 15 \mathrm{~kb}$ region of chromosome $3 \mathrm{~L}$ and encode the Para $\mathrm{Na}_{\mathrm{V}}$ channel auxiliary subunits, Teh2, Teh3, and TipE. Directly neighbouring the Teh 2 gene is a fourth homologue, Teh4, which, together with Teh1 on chromosome 3R, make up the full complement of five D. melanogaster TipE gene family members. The conservation of the Teh2-Teh3TipE synteny block across Insecta, despite generally high rates of genomic shuffling [20] and gene losses [23] over more than $\sim 350$ million years of evolution [24], suggests that strong constraints have maintained the local genomic architecture of the TipE locus (Figure 1).

Comparative analysis of the TipE gene family previously identified orthologues of $D$. melanogaster TipE, Teh1, Teh2, and Teh3 from A. gambiae, B. mori, and A. mellifera, as well as orthologues of all five family members from two other fruit flies, $D$. pseudoobscura and $D$. yakuba, and noted their similar genomic clustering [14]. Inspecting the genomic loci of all TipE gene family members in $D$. pulex and each of the 21 insect genomes facilitated comprehensive gene model curation to confirm and correct their predicted genomic architectures and protein-coding sequences (see Methods). These analyses identified the previously unannotated $A$. pisum TipE orthologue and the A. aegypti Teh1 orthologue, corrected the previously split gene model of the $A$. pisum Teh 2 orthologue, and improved several other gene model annotations (see Additional file 1, Table S1 for full details on all corrected gene models). The 


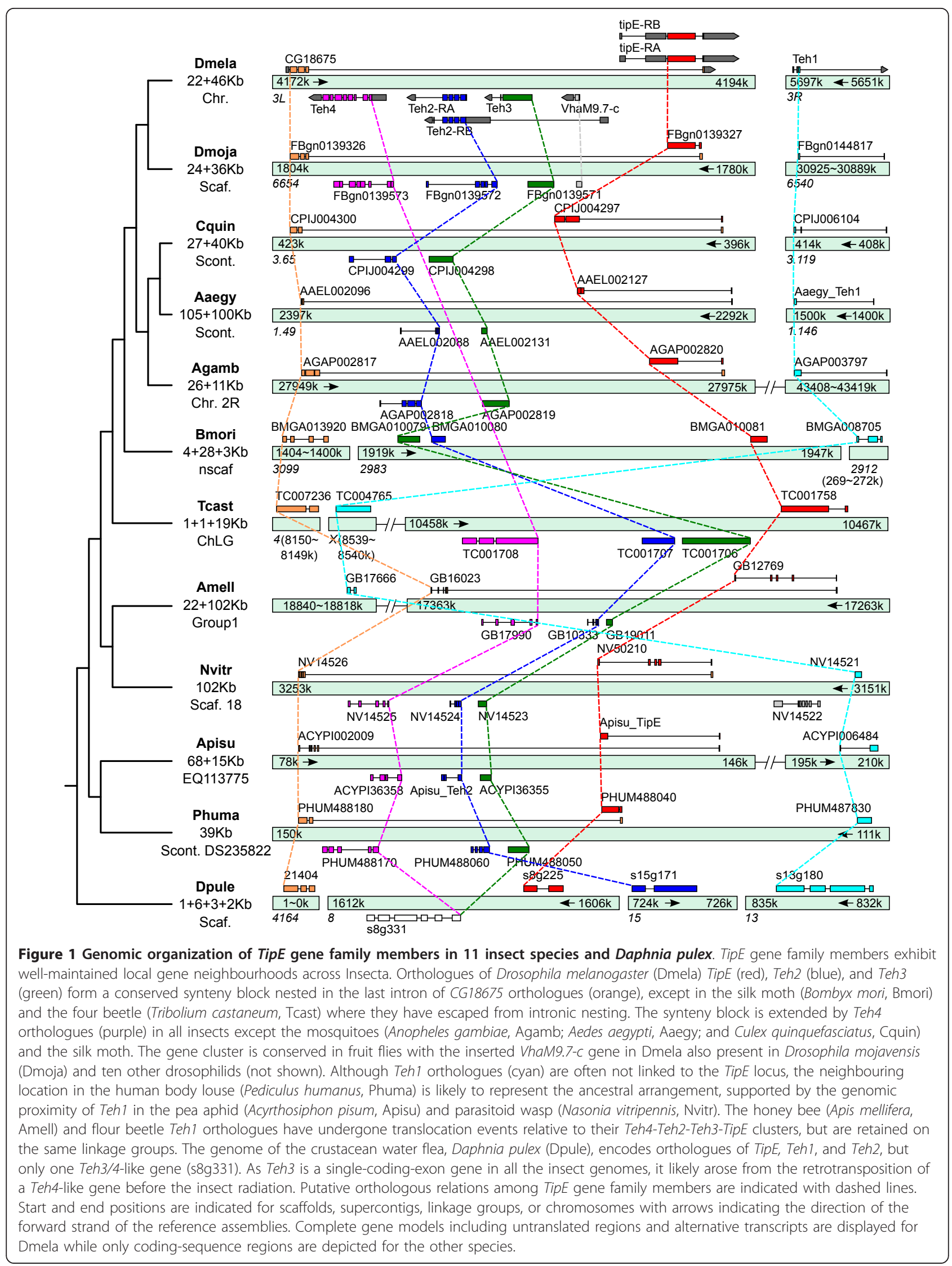


otherwise strictly-conserved arrangement of the Teh2Teh3-TipE synteny block, with Teh2 and Teh3 encoded on the opposite strand to TipE, is disrupted in the fruit flies by a short gene encoding a Vacuolar $\mathrm{H}^{+}$ATPase subunit (VhaM9.7-c in D. melanogaster) between Teh3 and TipE, and by a relative inversion of Teh2-Teh3 in the silk moth (Bmori). Teh4 orthologues are located adjacent to Teh 2 and extend the core synteny block in all the insects except the three mosquitoes and the silk moth, which appear to have lost the Teh4 gene. In the genome of the human body louse (Phuma), Teh1 is adjacent to TipE ( $\sim 15 \mathrm{~kb}$ apart), while in the pea aphid (Apisu) it is $\sim 63 \mathrm{~kb}$ away and separated by two intervening genes; in the parasitoid wasp (Nvitr), it is $\sim 24 \mathrm{~kb}$ away with only a single intervening gene. As the compact body louse genome is remarkably free from transposable elements and appears to have experienced less shuffling than other insect genomes [25], the gene cluster in the last common ancestor with all five TipE gene family members is likely to have resembled the genomic arrangement found in the body louse today.

\section{Structural constraints: intronic nesting of the TipE locus}

The evidence-based annotations of the D. melanogaster TipE locus provide a comprehensive view of the complex gene architectures and reveal that the coding sequences of this gene cluster - Teh4, Teh2, Teh3, VhaM9.7-c, and TipE - are all nested within the last intron of the neighbouring CG18675 gene (Figure 1). Such nested gene structures in eukaryotes are generally much more frequently gained than lost, suggesting that while intronic insertions may be relatively well-tolerated, deletions are more likely to be constrained due to their possible negative impact on both the nested and the host genes [26]. Single-copy orthologues of CG18675 can be identified in almost all sequenced metazoan genomes, as well as in some flagellate protozoa, green plants, and apicomplexan parasites, but appear to be absent from fungi and nematode worms. These gene sequences are relatively well-conserved, with $49 \%$ amino acid identity between CG18675 and the human orthologue (C21orf59, ENSG00000159079), and are matched by the sequence profile, domain of unknown function DUF2870 (IPR021298). Although it has been preserved as a single-copy gene across a broad phyletic range, the nesting of multiple genes within the last intron of CG18675 orthologues appears to have only occurred in insects. A genomic sequence gap obscures the start of the water flea (Dpule) CG18675 orthologue, nevertheless, the C-terminal coding exons align well with other CG18675 orthologues (see Additional file 2), and the last intron contains no nested genes. This suggests that while it is possible that water flea TipE-like genes escaped from intronic nesting, it is more likely that the nesting event occurred in the last common ancestor of insects, after the divergence from crustaceans.

The genomic span of insect CG18675 orthologues with nested TipE gene family members ranges from $~ 20$ $\mathrm{kb}$ in the fruit fly (Dmela) and the body louse (Phuma) to $\sim 100 \mathrm{~kb}$ in the Aedes mosquito (Aaegy), which, in addition to the insertion of the VhaM9.7-c gene in the drosophilid lineage, highlights the dynamic evolution of this region. Despite these perturbations, the intronic nesting and local gene arrangements of the TipE locus have remained largely intact. In addition to the constraint imposed by intronic nesting, a likely contributing factor is the overlap of the terminal exons of CG18675 and TipE. These exons contain both coding and untranslated regions, however, their overlapping coding regions are out of phase and encode two distinct terminal peptides of 37 (CG18675) and 20 (TipE) amino acids (see Additional file 3, Figure S1). Nesting of the TipE locus and the overlapping terminal exons are observed in the CG18675 orthologues from all the examined insect species except the flour beetle (Tcast) and the silk moth (Bmori). The flour beetle CG18657 orthologue (TC007236) is on a different linkage group to the TipE locus and the silk moth gene (BGIBMGA013920) is on a different scaffold. Both genes show conserved coding sequences that include the last coding exon, indicating that their gene models are complete (see Additional file 2), and their genomic spans of $\sim 2.5 \mathrm{~kb}$ (Bmori) and $\sim 1 \mathrm{~kb}$ (Tcast) are greatly reduced compared to other CG18675 orthologues. Thus, in these representative species from the Coleoptera and Lepidoptera lineages, the TipE locus appears to have been released from the constraint of intronic nesting. Despite their escape from intronic nesting, Teh2-Teh3 remain adjacent to TipE in the silk moth, and in the flour beetle the local gene arrangements of Teh4, Teh2, Teh3, and TipE are strictly maintained, suggesting possible functional constraints that preserve the architecture of the TipE locus.

\section{Functional constraints: coordinated regulation of TipE gene family members}

Intronic nesting may suggest possible mechanisms for coordinated regulation of host and nested genes, however, functional relations between host and their nested genes are usually unclear, and their expression profiles often exhibit inverse or no significant correlations $[26,27]$. Insect glucose-methanol-choline (GMC) oxidoreductase genes exhibit a similar nested genomic architecture to that of the TipE locus; most gene family members are encoded in a large intron of the flotillin-2 (Flo-2) gene in D. melanogaster, and its orthologues in other insects [20,28]. This cluster of GMC oxidoreductases may be coordinately regulated for developmental 
or physiological roles involved in ecdysteroid metabolism, which may be linked to the function of the host gene product, Flo-2, in lipid rafts [28]. Multiple nesting events of homologous genes within a single host intron could therefore facilitate coordinated regulation of the nested genes. Examining D. melanogaster gene expression data from different tissue samples (FlyAtlas [29]) and developmental series experiments (modENCODE [30]) revealed common and distinct spatial and temporal expression patterns of the TipE locus genes (see Additional file 4). Tissues with the highest transcript levels of TipE gene family members include the brain, thoracicoabdominal ganglion, eye, larval central nervous system, and the head, mirroring the expression of Para and consistent with their roles in excitable cells.

The developmental series data reveal two peaks of gene expression; the first, from the 14-16-hour embryo to the first larval stage (L1), is dominated by Teh4. The start of the second peak is evident from the 12-hour white prepupae, with maximal transcript levels at the 3day pupae and maintained expression in adult males, but not in adult females. TipE-Teh1 and Teh2-Teh3 are the best correlated pairs of expression profiles, TipE is also well-correlated with Teh2 and Teh4, but to a lesser extent with Teh3 (Spearman correlation test, Table 1). Thus, coordinated regulation appears not only amongst the intron-nested TipE gene family members, but also with the unlinked Teh1 gene. However, these coordinated expression profiles exhibit poor correlations with that of the host gene, CG18657, which displays a small peak during mid-embryogenesis and then increases again from the 12-hour L3 larval stage with greatly-

Table 1 Spearman correlation coefficient for gene expression data of $D$. melanogaster genes

\begin{tabular}{|c|c|c|c|c|c|c|c|}
\hline & TipE & Teh1 & Teh2 & Teh3 & Teh4 & Para & Slowpoke \\
\hline Teh1 & $\begin{array}{l}0.94 \\
* * *\end{array}$ & & & & & & \\
\hline Teh2 & $0.82 * *$ & $0.79 * *$ & & & & & \\
\hline Teh3 & $0.70 *$ & $0.69 *$ & $\begin{array}{l}0.94 \\
* * *\end{array}$ & & & & \\
\hline Teh4 & $0.80^{* *}$ & $0.71 *$ & $0.59 *$ & $\begin{array}{l}0.52 \\
\text { NS }\end{array}$ & & & \\
\hline Para & $\begin{array}{l}0.92 \\
* * *\end{array}$ & $\begin{array}{l}0.90 \\
* * *\end{array}$ & $0.77 * *$ & $0.61 *$ & $0.69 *$ & & \\
\hline Slowpoke & $\begin{array}{l}0.92 \\
* * *\end{array}$ & $\begin{array}{l}0.92 \\
* * *\end{array}$ & $0.81 *$ & $0.71 *$ & $0.66 *$ & $\begin{array}{l}0.89 \\
* * *\end{array}$ & \\
\hline CG18675 & $\begin{array}{l}0.42 \\
\text { NS }\end{array}$ & $\begin{array}{l}0.41 \\
\text { NS }\end{array}$ & $\begin{array}{l}0.44 \\
\text { NS }\end{array}$ & $\begin{array}{l}0.51 \\
\text { NS }\end{array}$ & $\begin{array}{l}0.34 \\
\text { NS }\end{array}$ & $\begin{array}{l}0.21 \\
\text { NS }\end{array}$ & 0.47 NS \\
\hline
\end{tabular}

Spearman correlation coefficient p-values: ${ }^{* *}<1 \mathrm{e}-09,{ }^{* *}<1 \mathrm{e}-06,{ }^{*}<1 \mathrm{e}-03$, NS not significant

Spearman correlation tests performed on $D$. melanogaster gene expression data from developmental series experiments (see Additional file 4) using $R$ (http://www.r-project.org/) show that the expression profiles of TipE gene family members are well-correlated, as well as with Para and Slowpoke.

However, the expression profiles of these genes exhibit poor correlation with the host gene of TipE gene cluster, CG18675. elevated expression in adult males and almost undetectable levels in adult females, consistent with the high transcript levels observed in testis samples. In contrast, the TipE gene family members' expression profiles are much better-matched to that of Para, particularly TipE and Teh1 - these two members also induced the largest increases of expression levels of Para $\mathrm{Na}_{\mathrm{V}}$ channels in Xenopus oocytes [14]. Thus, D. melanogaster TipE gene family members exhibit coordinated spatial and temporal regulation that also correlates well with the expression of Para, their functional $\mathrm{Na}_{\mathrm{V}}$ channel partner, but which appears distinct from the host gene, CG18657.

\section{Evolutionary history of the insect TipE gene family}

Phylogenetic analysis of TipE gene family members from $D$. pulex and a representative subset of the 21 insect species highlights the five groups of orthologues (see Figure 2 and Additional file 3, Figure S2). The TipETeh1-Teh2 proteins form a distinct clade from Teh3Teh4, suggesting possible functional divergence between members of these two clades. This is supported by the presence of the epidermal growth factor (EGF)-like domains in the extracellular loop regions of Teh3 and Teh4 proteins that are not found in other TipE gene family members, which may interact with extracellular matrix components [14]. Conserved sequence motif analysis using MEME [31] supports the orthology assignments, highlights the extended extracellular loop regions of the Teh 3 and Teh4 orthologues, and shows that the sequence regions common to all TipE gene family members are comprised of the two transmembrane domains and parts of the extracellular loop (see Additional file 3, Figure S3). These regions exhibit high rates of amino acid substitutions, and this variation may explain the lack of confident bootstrap support for several nodes on the TipE gene family phylogeny (see Figure 2 and Additional file 3, Figure S2). The D. pulex genome encodes only one Teh3/4-like gene and Teh3 is found as a single-coding-exon gene in all the examined insect genomes; the alignment of the D. melanogaster genes identifies seven sites with uninterrupted coding sequence in Teh3 but with introns in Teh4, further suggesting that Teh3 arose from the retrotransposition of a Teh4-like gene of the last common ancestor before the insect radiation. In the silk moth and the three mosquitoes, the absence of Teh4 orthologues implies that the Teh3 retrogene, which integrated into the same local genomic environment, could have assumed the functions of the original multi-exon ancestor. The D. pulex TipE orthologue is resolved on the TipE gene family phylogeny (Figure 2), and although Teh1 and Teh2 orthologues are not confidently resolved, they can be identified by comparing their motif architectures (see 
Additional file 3, Figure S3) with the insect Teh1 and Teh2 orthologues. Thus, it is likely that the ancestral pancrustacean genome encoded four TipE gene family members, the subsequent retrotransposition of Teh4 after the divergence of the crustacean and insect lineages gave rise to Teh3 and created a possible functional redundancy which led to the loss of Teh4 in the silk moth and the mosquitoes.

Performing BLAST and PSI-BLAST homology searches of non-redundant sequence databases using TipE gene family members failed to identify any significant matches beyond Pancrustacea. Orthologues of Para are present in all the examined insects and the water flea, as well as in the closest outgroup species, the arachnid deer tick, Ixodes scapularis (see Additional file 1, Table S1 and Additional file 3, Figure S4), however, no TipE homologues were identified in either the deer tick's assembled scaffolds or sequence trace archives. Similarly, TipE-like auxiliary beta-subunits are not found in the genome of the nematode worm, Caenorhabditis elegans, which also lacks any known $\mathrm{Na}_{\mathrm{V}}$ channel homologues, despite the likely presence of an ancestral sodium channel before the divergence of vertebrates and invertebrates [32]. Scans with Hidden Markov Model (HMM) profiles built from multiple protein sequence alignments of TipE gene family members (see Methods) also produced no significant matches to tick or worm proteins. However, the improved search sensitivity of the HMM profiles revealed distant homology with beta-4 auxiliary subunits of vertebrate big-conductance calcium-activated potassium $\left(\mathrm{BK}_{\mathrm{Ca}}\right)$ channels (KCNMB4, a member of the Slo-beta or BK-beta subunit family): the HMM profile built from the 32 TipE gene family members shown in Additional file 3, Figure S2 identifies human, mouse, cow, and frog KCNMB4 proteins with e-values of less than 1e-05. A reciprocal homology search of arthropod proteomes with a vertebrate KCNMB4 HMM profile identifies insect Teh1 orthologues with e-values of less than 1e-03. A notable difference occurs in the extracellular loop region of TipE, Teh3, and Teh4 proteins, which are longer than the loops in Slo-beta proteins and exhibit EGF-like domains in Teh3 and Teh4 [2,14]. Although the possibility of convergent evolution of these two families of auxiliary subunits cannot be excluded, the similar protein architecture of TipE-like and Slo-beta proteins, with two hydrophobic regions that anchor an extracellular loop containing putative disulphide bridges, further supports their common origin.

Their likely common ancestry with Slo-beta subunits suggests that some TipE gene family members may also play a role in the regulation of $\mathrm{BK}_{\mathrm{Ca}}$ channels. This type of ion channel is found across metazoan species, and is named Slowpoke in D. melanogaster. Although Slowpoke exhibits generally higher expression levels than Para, particularly in the eye, head, and spermatheca, their developmental expression profiles are well-correlated and match those of the TipE gene family members (see Table 1 and Additional file 4). However, rather than suggesting a role for some TipE gene family members in the regulation of Slowpoke, their similar developmental expression profiles may arise simply because both channels function in excitable cells. Indeed, TipE gene family members showed no effects on Slowpoke potassium currents in D. melanogaster cell lines [14], which are instead regulated by an auxiliary subunit with a protein kinase-like domain, Slowpoke binding protein [33]. The likely common ancestor of the TipE-like and Slo-beta auxiliary subunits may therefore have functioned together with the pore-forming alpha-subunits of an ancestral metazoan potassium or sodium channel, subsequently undergoing independent fine-tuning to control calcium-activated potassium currents in vertebrates and voltage-gated sodium currents in the invertebrate lineage.

\section{Conclusion}

The TipE gene family of Para $\mathrm{Na}_{\mathrm{V}}$ channel auxiliary subunits forms a remarkably conserved genomic cluster of orthologues with local gene arrangements maintained across many diverse insect species. This conservation suggests strong constraints that preserve the organisation of the TipE gene cluster, as observed for the wellstudied Hox gene clusters that nevertheless appear to tolerate limited gene rearrangements in Drosophila [34]. Comparing the TipE synteny block architecture across 21 insect species and examining expression patterns of D. melanogaster genes provides evidence of both structural and functional constraints that preserve the TipE cluster. The ancestral insect TipE gene cluster is likely to have resembled the genomic arrangement found in the human body louse today; the TipE, Teh2, Teh3, and Teh4 genes nested within the last intron of the CG18657 ancestor, and directly neighbouring Teh1. Although no TipE-like genes were identified in arachnid or nematode genomes, homologues encoded in the $D$. pulex genome suggest an ancient pancrustacean repertoire of four TipE gene family members. Retrotransposition of Teh 4 gave rise to the insect Teh3 retrogene following the divergence of crustaceans and insects, which may have generated functional redundancy that allowed for the loss of Teh4 in the silk moth and the mosquitoes. Local gene arrangements of the TipE locus are well-maintained despite the apparent escape from intronic nesting in the silk moth and the flour beetle genomes, suggesting additional constraints that preserve the genomic architecture. D. melanogaster gene expression data reveal coordinated spatial and temporal 
regulation of the TipE gene family members that is distinct from the host gene, but which correlates well with the expression of their regulatory target, Para. Such functional constraints are likely to contribute to the preservation of the TipE synteny block across Insecta, to maintain coordinated expression of gene family members with or without the genomic constraint of intronic nesting. Examining the insect TipE gene family reveals several key evolutionary events that characterize the evolution of genes within their genomic contexts: intronic nesting, escape from nesting, overlapping exons, retrotransposition, translocation, gene loss, coordinated regulation, and conserved synteny. Exploration of the evolutionary histories of genes maintained in such synteny blocks therefore offers the opportunity to identify putative structural and functional constraints on gene rearrangements and their relative contributions to genome evolution.

The common ancestry of the TipE-like and Slo-beta auxiliary subunits suggests a remarkable plasticity in the evolution of mechanisms for regulating ion channel activities. An ancestral ion channel auxiliary subunit appears to have evolved to regulate calcium-activated potassium currents in vertebrates and voltage-gated sodium currents in the invertebrate lineage. Thus, although the pore-forming alpha-subunits are generally well-conserved across metazoan species, their regulatory partners appear to exhibit more lineage-specific functions. Consequently, targeting the activity of such auxiliary subunits may prove a successful strategy for the development of insecticides that are not toxic to vertebrates, and a more detailed understanding of their molecular functions in different insect species may facilitate targeted interventions that minimise ecological knockon effects. As well as regulating functional expression and gating kinetics of ion channels, auxiliary subunits are known to influence interactions with naturallyoccurring toxins, such as scorpion venom peptides which inhibit big-conductance calcium channels [35]. Following Nature's lead could therefore offer novel prospects for the development of insect control strategies that target ion channel activities.

\section{Methods}

\section{Genomic resources}

Official gene sets and assembled genome sequences for 23 arthropod species were retrieved from publically available resources including AphidBase [36], BeetleBase [37], FlyBase [38], Hymenoptera Genome Database [39], SilkDB [40], VectorBase [41], and wFleaBase [42]; see Additional file 1, Table S2 for full details. The examined arthropod species include: Diptera - Drosophila melanogaster (Dmela) [43] and 11 other fruit flies [44]: Drosophila sechellia (Dsech), Drosophila simulans (Dsimu),
Drosophila erecta (Derec), Drosophila yakuba (Dyaku), Drosophila ananassae (Danan), Drosophila persimilis (Dpers), Drosophila pseudoobscura (Dpseu), Drosophila willistoni (Dwill), Drosophila mojavensis (Dmoja), Drosophila virilis (Dviri), Drosophila grimshawi (Dgrim), as well as three mosquitoes: Anopheles gambiae (Agamb) [45], Aedes aegypti (Aaegy) [46], Culex quinquefasciatus (Cquin) [47]; Lepidoptera - the silk moth, Bombyx mori (Bmori) [48]; Coleoptera - the flour beetle, Tribolium castaneum (Tcast) [49]; Hymenoptera - the honey bee, Apis mellifera (Amell) [50] and the parasitoid wasp, Nasonia vitripennis (Nvitr) [51]; Hemiptera - the pea aphid, Acyrthosiphon pisum (Apisu) [52]; Phthiraptera the human body louse, Pediculus humanus (Phuma) [25]; Crustacea - the water flea, Daphnia pulex (Dpule) [53]; and Arachnida - the deer tick, Ixodes scapularis (Iscap).

\section{Synteny block identification}

The conserved genomic cluster of TipE gene family members was detected by employing synteny block identification procedures developed from a strategy based on orthologous gene anchors [20,54]. Initial pairwise species comparisons used single-copy orthologous genes from the OrthoDB $[55,56]$, requiring at least two pairs of neighbouring orthologues and allowing one intervening orthologue at most. Subsequently, synteny block extension across multiple species was performed by successive projections of pairwise blocks across the species phylogeny.

\section{Gene model curation}

Protein sequence comparisons of TipE gene family members and orthologues of CG18675 revealed several annotation errors including missing exons and split gene models. Gene model curation employed additional available information such as expressed sequenced tags (ESTs) and homology-based gene predictions using Fgenesh+ [57] and Wise2 [58] to confirm and correct the annotated gene models. HMM profiles of TipE gene family members and CG18675 orthologues used for gene predictions were built using HMMER [59]. The HMMs were also used to scan the proteomes of additional non-arthropod species including Caenorhabditis elegans, Nematostella vectensis, Hydra magnipapillata, Strongylocentrotus purpuratus, Branchiostoma floridae, Danio rerio, Xenopus tropicalis, Gallus gallus, Bos taurus, Mus musculus and Homo sapiens. Megablast and tBLASTn homology searches against the unassembled sequence trace archives, as well as the assembled genomes, were performed respectively to check for the presence of any additional genes potentially missed by the assembly or annotation procedures. 


\section{Phylogenetic analyses}

Multiple protein sequence alignments of TipE gene family members and CG18675 orthologues were built using MUSCLE [60], and confidently aligned regions were extracted with Gblocks [61]. Maximum likelihood phylogenetic trees were reconstructed using PhyML [62], with the Jones-Taylor-Thornton (JTT) substitution model, four relative substitution rate categories, estimated proportions of invariable sites and gamma distribution parameters, and 100 bootstrap samples. To support the phylogenetic analyses, conserved motif analysis of the protein sequences of TipE gene family members was performed using MEME [31], with the motif width from 6 to 50 amino acids and a maximum of 10 motifs.

\section{Additional material}

Additional file 1: Genomic resources and gene information. Table S1: Details of the genes included in this study; Table S2: Details of the genomic resources used in this study.

Additional file 2: CG18675 MSA. Multiple sequence alignment of CG18675 orthologues.

Additional file 3: Additional Figures. Figure S1: Detailed map of the last exons of CG18675 and TipE in Drosophila melanogaster; Figure S2: Phylogenetic tree of TipE gene family members in six insect species and Daphnia pulex; Figure S3: Motif architectures of TipE-like proteins in six insect species and Daphnia pulex; Figure S4: Phylogenetic tree of Para orthologues in 11 insect species, Daphnia pulex and Ixodes scapularis

Additional file 4: Gene expression data. High-throughput gene expression data of $D$. melanogaster genes.

\section{List of abbreviations}

BK-beta: big-conductance calcium-activated potassium channel beta subunit; $\mathrm{BK}_{\mathrm{Ca}}$ channel: big-conductance calcium-activated potassium channel; DSC1: Drosophila sodium channel 1; EGF: epidermal growth factor; Flo-2: flotillin-2; GMC: glucose-methanol-choline; HMM: Hidden Markov Model; JTT: JonesTaylor-Thornton; KCNMB4: calcium-activated potassium channel subunit beta-4; Nav channel: voltage-gated sodium channel; Para: paralytic; Slo-beta: Slowpoke-beta; Teh1-4: TipE homolog 1-4; TipE: temperature-induced paralytic E; VhaM9.7-c: Vacuolar $\mathrm{H}^{+}$ATPase subunit M9.7-c.

\section{Acknowledgements}

The authors would like to thank Dr Evgenia Kriventseva, Dr Thomas Junier and all members of the Computational Evolutionary Genomics Group for useful suggestions and discussions. This work was supported by the Swiss National Science Foundation (3100A0-112588).

\section{Author details}

'Department of Genetic Medicine and Development, University of Geneva Medical School, rue Michel-Servet 1, 1211 Geneva, Switzerland. ${ }^{2}$ Swiss Institute of Bioinformatics, rue Michel-Servet 1, 1211 Geneva, Switzerland. ${ }^{3}$ Imperial College London, South Kensington Campus, London, SW7 2AZ, UK.

\section{Authors' contributions}

$J \mathrm{~L}, \mathrm{RMW}$ and EMZ conceived and designed the study, and wrote the manuscript. JL performed the computational data analyses. All authors read and approved the final manuscript.

\section{Competing interests}

The authors declare that they have no competing interests.
Received: 13 July 2011 Accepted: 18 November 2011

Published: 18 November 2011

\section{References}

1. Widmark J, Sundström G, Ocampo Daza D, Larhammar D: Differential Evolution of Voltage-Gated Sodium Channels in Tetrapods and Teleost Fishes. Molecular Biology and Evolution 2011, 28:859-871.

2. Tseng TT, McMahon AM, Johnson VT, Mangubat EZ, Zahm RJ, Pacold ME, Jakobsson E: Sodium channel auxiliary subunits. J Mol Microbiol Biotechnol 2007, 12:249-262.

3. Kulkarni NH, Yamamoto AH, Robinson KO, Mackay TFC, Anholt RRH: The DSC1 channel, encoded by the smi60E locus, contributes to odorguided behavior in Drosophila melanogaster. Genetics 2002, 161:1507-1516.

4. Zhou W, Chung I, Liu Z, Goldin AL, Dong K: A voltage-gated calciumselective channel encoded by a sodium channel-like gene. Neuron 2004, 42:101-112.

5. Suzuki DT, Grigliatti T, Williamson R: Temperature-sensitive mutations in Drosophila melanogaster. VII. A mutation (para-ts) causing reversible adult paralysis. Proc Natl Acad Sci USA 1971, 68:890-893.

6. Siddiqi O, Benzer S: Neurophysiological defects in temperature-sensitive paralytic mutants of Drosophila melanogaster. Proc Natl Acad Sci USA 1976, 73:3253-3257.

7. Wu CF, Ganetzky B: Genetic alteration of nerve membrane excitability in temperature-sensitive paralytic mutants of Drosophila melanogaster. Nature 1980, 286:814-816.

8. Loughney K, Kreber R, Ganetzky B: Molecular analysis of the para locus, a sodium channel gene in Drosophila. Cell 1989, 58:1143-1154.

9. Jackson FR, Wilson SD, Hall LM: The tip-E mutation of Drosophila decreases saxitoxin binding and interacts with other mutations affecting nerve membrane excitability. I Neurogenet 1986, 3:1-17.

10. Ganetzky B: Neurogenetic analysis of Drosophila mutations affecting sodium channels: synergistic effects on viability and nerve conduction in double mutants involving tip-E. J Neurogenet 1986, 3:19-31.

11. Feng G, Deák P, Chopra M, Hall LM: Cloning and functional analysis of TipE, a novel membrane protein that enhances Drosophila para sodium channel function. Cell 1995, 82:1001-1011.

12. Hodges DD, Lee D, Preston CF, Boswell K, Hall LM, O'Dowd DK: tipE Regulates Na+-dependent Repetitive Firing in Drosophila Neurons. Molecular and Cellular Neuroscience 2002, 19:402-416.

13. Warmke JW, Reenan RAG, Wang P, Qian S, Arena JP, Wang J, Wunderler $D$, Liu K, Kaczorowski GJ, der Ploeg LHTV, Ganetzky B, Cohen CJ: Functional Expression of Drosophila para Sodium Channels. The Journal of General Physiology 1997, 110:119-133.

14. Derst C, Walther C, Veh RW, Wicher D, Heinemann SH: Four novel sequences in Drosophila melanogaster homologous to the auxiliary Para sodium channel subunit TipE. Biochem Biophys Res Commun 2006, 339:939-948.

15. Dong K: Insect sodium channels and insecticide resistance. Invert Neurosci 2007, 7:17-30.

16. Davies TGE, Field LM, Usherwood PNR, Williamson MS: DDT, pyrethrins, pyrethroids and insect sodium channels. IUBMB Life 2007, 59:151-162.

17. Zlotkin E: The insect voltage-gated sodium channel as target of insecticides. Annu Rev Entomol 1999, 44:429-455.

18. Vais H, Williamson MS, Devonshire AL, Usherwood PN: The molecular interactions of pyrethroid insecticides with insect and mammalian sodium channels. Pest Manag Sci 2001, 57:877-888.

19. Soderlund DM, Knipple DC: The molecular biology of knockdown resistance to pyrethroid insecticides. Insect Biochem Mol Biol 2003, 33:563-577.

20. Zdobnov EM, Bork P: Quantification of insect genome divergence. Trends Genet 2007, 23:16-20

21. Garcia-Fernàndez J: The genesis and evolution of homeobox gene clusters. Nat Rev Genet 2005, 6:881-892.

22. Cho SJ, Vallès Y, Giani VC Jr, Seaver EC, Weisblat DA: Evolutionary dynamics of the wnt gene family: a lophotrochozoan perspective. Mol Biol Evol 2010, 27:1645-1658.

23. Wyder S, Kriventseva EV, Schröder R, Kadowaki T, Zdobnov EM: Quantification of ortholog losses in insects and vertebrates. Genome Biol 2007, 8:R242.

24. Hedges SB, Kumar S: The timetree of life Oxford University Press; 2009. 
25. Kirkness EF, Haas BJ, Sun W, Braig HR, Perotti MA, Clark JM, Lee SH, Robertson HM, Kennedy RC, Elhaik E, Gerlach D, Kriventseva EV, Elsik CG, Graur D, Hill CA, Veenstra JA, Walenz B, Tubío JMC, Ribeiro JMC, Rozas J, Johnston JS, Reese JT, Popadic A, Tojo M, Raoult D, Reed DL, Tomoyasu Y, Krause E, Mittapalli O, Margam VM, et al: Genome sequences of the human body louse and its primary endosymbiont provide insights into the permanent parasitic lifestyle. Proceedings of the National Academy of Sciences 2010, 107:12168-12173.

26. Assis R, Kondrashov AS, Koonin EV, Kondrashov FA: Nested genes and increasing organizational complexity of metazoan genomes. Trends in Genetics 2008, 24:475-478.

27. Kumar A: An Overview of Nested Genes in Eukaryotic Genomes. Eukaryot Cell 2009, 8:1321-1329.

28. lida K, Cox-Foster D, Yang X, Ko WY, Cavener D: Expansion and evolution of insect GMC oxidoreductases. BMC Evolutionary Biology 2007, 7:75.

29. Chintapalli VR, Wang J, Dow JAT: Using FlyAtlas to identify better Drosophila melanogaster models of human disease. Nat Genet 2007, 39:715-720.

30. Celniker SE, Dillon LAL, Gerstein MB, Gunsalus KC, Henikoff S, Karpen GH, Kellis M, Lai EC, Lieb JD, MacAlpine DM, Micklem G, Piano F, Snyder M, Stein L, White KP, Waterston RH: Unlocking the secrets of the genome. Nature 2009, 459:927-930.

31. Bailey $\mathrm{TL}$, Elkan C: Fitting a mixture model by expectation maximization to discover motifs in biopolymers. Proc Int Conf Intell Syst Mol Biol 1994, 2:28-36.

32. Yu FH, Catterall WA: Overview of the voltage-gated sodium channel family. Genome Biol 2003, 4:207.

33. Ma H, Zhang J, Levitan IB: Slob, a Slowpoke channel-binding protein, modulates synaptic transmission. The Journal of General Physiology 2011, 137:225-238

34. Negre B, Ruiz A: HOM-C evolution in Drosophila: is there a need for Hox gene clustering? Trends Genet 2007, 23:55-59.

35. Berkefeld H, Fakler B, Schulte U: Ca2+-Activated K+ Channels: From Protein Complexes to Function. Physiological Reviews 2010, 90:1437-1459.

36. Gauthier JP, Legeai F, Zasadzinski A, Rispe C, Tagu D: AphidBase: a database for aphid genomic resources. Bioinformatics 2007, 23:783-784.

37. Kim HS, Murphy T, Xia J, Caragea D, Park Y, Beeman RW, Lorenzen MD, Butcher S, Manak JR, Brown SJ: BeetleBase in 2010: revisions to provide comprehensive genomic information for Tribolium castaneum. Nucleic Acids Research 2009, 38:D437-D442.

38. Tweedie S, Ashburner M, Falls K, Leyland P, McQuilton P, Marygold S, Millburn G, Osumi-Sutherland D, Schroeder A, Seal R, Zhang H, The FlyBase Consortium: FlyBase: enhancing Drosophila Gene Ontology annotations. Nucleic Acids Research 2009, 37:D555-D559

39. Munoz-Torres MC, Reese JT, Childers CP, Bennett AK, Sundaram JP, Childs KL, Anzola JM, Milshina N, Elsik CG: Hymenoptera Genome Database: integrated community resources for insect species of the order Hymenoptera. Nucleic Acids Research 2010, 39:D658-D662.

40. Wang J, Xia Q, He X, Dai M, Ruan J, Chen J, Yu G, Yuan H, Hu Y, Li R, Feng T, Ye C, Lu C, Wang J, Li S, Wong GK-S, Yang H, Wang J, Xiang Z, Zhou Z, Yu J: SilkDB: a knowledgebase for silkworm biology and genomics. Nucleic Acids Res 2005, 33:D399-402.

41. Lawson D, Arensburger P, Atkinson P, Besansky NJ, Bruggner RV, Butler R, Campbell KS, Christophides GK, Christley S, Dialynas E, Hammond M, Hill CA, Konopinski N, Lobo NF, MacCallum RM, Madey G, Megy K, Meyer J, Redmond S, Severson DW, Stinson EO, Topalis P, Birney E, Gelbart WM, Kafatos FC, Louis C, Collins FH: VectorBase: a data resource for invertebrate vector genomics. Nucleic Acids Research 2009, 37:D583-D587.

42. Colbourne J, Singan V, Gilbert D: wFleaBase: the Daphnia genome database. BMC Bioinformatics 2005, 6:45

43. Adams MD, Celniker SE, Holt RA, Evans CA, Gocayne JD, Amanatides PG, Scherer SE, Li PW, Hoskins RA, Galle RF, George RA, Lewis SE, Richards S, Ashburner M, Henderson SN, Sutton GG, Wortman JR, Yandell MD, Zhang Q, Chen LX, Brandon RC, Rogers YH, Blazej RG, Champe M, Pfeiffer BD, Wan KH, Doyle C, Baxter EG, Helt G, Nelson CR, et al: The Genome Sequence of Drosophila melanogaster. Science 2000, 287:2185-2195.

44. Clark AG, Eisen MB, Smith DR, Bergman CM, Oliver B, Markow TA, Kaufman TC, Kellis M, Gelbart W, lyer VN, Pollard DA, Sackton TB, Larracuente AM, Singh ND, Abad JP, Abt DN, Adryan B, Aguade M, Akashi $\mathrm{H}$, Anderson WW, Aquadro CF, Ardell DH, Arguello R, Artieri CG,
Barbash DA, Barker D, Barsanti P, Batterham P, Batzoglou S, Begun D, et al Evolution of genes and genomes on the Drosophila phylogeny. Nature 2007, 450:203-218.

45. Holt RA, Subramanian GM, Halpern A, Sutton GG, Charlab R, Nusskern DR, Wincker P, Clark AG, Ribeiro JM, Wides R, Salzberg SL, Loftus B, Yandell M, Majoros WH, Rusch DB, Lai Z, Kraft CL, Abril JF, Anthouard V, Arensburger P, Atkinson PW, Baden $H$, de Berardinis V, Baldwin D, Benes V, Biedler J, Blass C, Bolanos R, Boscus D, Barnstead M, et al: The genome sequence of the malaria mosquito Anopheles gambiae. Science 2002, 298:129-149.

46. Nene V, Wortman JR, Lawson D, Haas B, Kodira C, Tu ZJ, Loftus B, Xi Z, Megy K, Grabherr M, Ren Q, Zdobnov EM, Lobo NF, Campbell KS, Brown SE, Bonaldo MF, Zhu J, Sinkins SP, Hogenkamp DG, Amedeo P, Arensburger P, Atkinson PW, Bidwell S, Biedler J, Birney E, Bruggner RV, Costas J, Coy MR, Crabtree J, Crawford M, et al: Genome sequence of Aedes aegypti, a major arbovirus vector. Science 2007, 316:1718-1723.

47. Arensburger P, Megy K, Waterhouse RM, Abrudan J, Amedeo P, Antelo B, Bartholomay L, Bidwell S, Caler E, Camara F, Campbell CL, Campbell KS, Casola C, Castro MT, Chandramouliswaran I, Chapman SB, Christley S, Costas J, Eisenstadt E, Feschotte C, Fraser-Liggett C, Guigo R, Haas B, Hammond M, Hansson BS, Hemingway J, Hill SR, Howarth C, Ignell R, Kennedy RC, et al: Sequencing of Culex quinquefasciatus Establishes a Platform for Mosquito Comparative Genomics. Science 2010, 330:86-88

48. Xia Q, Zhou Z, Lu C, Cheng D, Dai F, Li B, Zhao P, Zha X, Cheng T, Chai C, Pan G, Xu J, Liu C, Lin Y, Qian J, Hou Y, Wu Z, Li G, Pan M, Li C, Shen Y, Lan X, Yuan L, Li T, Xu H, Yang G, Wan Y, Zhu Y, Yu M, Shen W, et al: A draft sequence for the genome of the domesticated silkworm (Bombyx mori). Science 2004, 306:1937-1940.

49. Richards S, Gibbs RA, Weinstock GM, Brown SJ, Denell R, Beeman RW, Gibbs R, Beeman RW, Brown SJ, Bucher G, Friedrich M, Grimmelikhuijzen CJ Klingler M, Lorenzen M, Richards S, Roth S, Schröder R, Tautz D, Zdobnov EM, Muzny D, Gibbs RA, Weinstock GM, Attaway T, Bell S, Buhay CJ, Chandrabose MN, Chavez D, Clerk-Blankenburg KP, Cree A, Dao M, et al: The genome of the model beetle and pest Tribolium castaneum. Nature 2008, 452:949-955.

50. Weinstock GM, Robinson GE, Gibbs RA, Weinstock GM, Robinson GE, Worley KC, Evans JD, Maleszka R, Robertson HM, Weaver DB, Beye M, Bork P, Elsik CG, Evans JD, Hartfelder K, Hunt GJ, Robertson HM, Robinson GE, Maleszka R, Weinstock GM, Worley KC, Zdobnov EM, Hartfelder K, Amdam GV, Bitondi MM, Collins AM, Cristino AS, Evans JD, Lattorff MG, Lobo $\mathrm{CH}$, et al: Insights into social insects from the genome of the honeybee Apis mellifera. Nature 2006, 443:931-949.

51. Werren JH, Richards S, Desjardins CA, Niehuis O, Gadau J, Colbourne JK, Beukeboom LW, Desplan C, Elsik CG, Grimmelikhuijzen CJ, Kitts P, Lynch JA, Murphy T, Oliveira DC, Smith CD, van de Zande L, Worley KC, Zdobnov EM, Aerts M, Albert S, Anaya VH, Anzola JM, Barchuk AR, Behura SK, Bera AN, Berenbaum MR, Bertossa RC, Bitondi MM, Bordenstein SR, Bork P, et al: Functional and evolutionary insights from the genomes of three parasitoid Nasonia species. Science 2010, 327:343-348.

52. Richards S, Gibbs RA, Gerardo NM, Moran N, Nakabachi A, Richards S, Stern D, Tagu D, Wilson AC, Muzny D, Kovar C, Cree A, Chacko J, Chandrabose MN, Dinh HH, Gabisi RA, Hines S, Hume J, Jhangian SN, Joshi V, Lewis LR, Liu YS, Lopez J, Morgan MB, Nguyen NB, Okwuonu GO, Ruiz SJ, Santibanez J, Wright RA, Fowler GR, et al: Genome sequence of the pea aphid Acyrthosiphon pisum. PLOS Biol 2010, 8:e1000313.

53. Colbourne JK, Pfrender ME, Gilbert D, Thomas WK, Tucker A, Oakley TH, Tokishita S, Aerts A, Arnold GJ, Basu MK, Bauer DJ, Cáceres CE, Carmel L, Casola C, Choi JH, Detter JC, Dong Q, Dusheyko S, Eads BD, Fröhlich T, Geiler-Samerotte KA, Gerlach D, Hatcher P, Jogdeo S, Krijgsveld J, Kriventseva EV, Kültz D, Laforsch C, Lindquist E, Lopez J, et al: The ecoresponsive genome of Daphnia pulex. Science 2011, 331:555-561.

54. Zdobnov EM, von Mering C, Letunic I, Torrents D, Suyama M, Copley RR, Christophides GK, Thomasova D, Holt RA, Subramanian GM, Mueller HM, Dimopoulos G, Law JH, Wells MA, Birney E, Charlab R, Halpern AL, Kokoza E, Kraft CL, Lai Z, Lewis S, Louis C, Barillas-Mury C, Nusskern D, Rubin GM, Salzberg SL, Sutton GG, Topalis P, Wides R, Wincker P, et al: Comparative Genome and Proteome Analysis of Anopheles gambiae and Drosophila melanogaster. Science 2002, 298:149-159.

55. Kriventseva EV, Rahman N, Espinosa O, Zdobnov EM: OrthoDB: the hierarchical catalog of eukaryotic orthologs. Nucleic Acids Research 2008, 36:D271-D275. 
56. Waterhouse RM, Zdobnov EM, Tegenfeldt F, Li J, Kriventseva EV: OrthoDB: the hierarchical catalog of eukaryotic orthologs in 2011. Nucleic Acids Research 2011, 39:D283-D288.

57. Salamov AA, Solovyev W: Ab initio Gene Finding in Drosophila Genomic DNA. Genome Research 2000, 10:516-522.

58. Birney E, Clamp M, Durbin R: GeneWise and Genomewise. Genome Research 2004, 14:988-995.

59. Eddy SR: Profile hidden Markov models. Bioinformatics 1998, 14:755-763.

60. Edgar RC: MUSCLE: multiple sequence alignment with high accuracy and high throughput. Nucleic Acids Research 2004, 32:1792-1797.

61. Castresana J: Selection of Conserved Blocks from Multiple Alignments for Their Use in Phylogenetic Analysis. Molecular Biology and Evolution 2000, 17:540-552.

62. Guindon S, Gascuel O: A Simple, Fast, and Accurate Algorithm to Estimate Large Phylogenies by Maximum Likelihood. Systematic Biology 2003, 52:696-704.

doi:10.1186/1471-2148-11-337

Cite this article as: Li et al:: A remarkably stable TipE gene cluster: evolution of insect Para sodium channel auxiliary subunits. BMC Evolutionary Biology 2011 11:337.

\section{Submit your next manuscript to BioMed Central} and take full advantage of:

- Convenient online submission

- Thorough peer review

- No space constraints or color figure charges

- Immediate publication on acceptance

- Inclusion in PubMed, CAS, Scopus and Google Scholar

- Research which is freely available for redistribution

Submit your manuscript at www.biomedcentral.com/submit 